\title{
Combined sarcomatoid hepatocellular and cholangiocarcinoma: a case report and literature review
}

\author{
Julian Shun Tsang1, Kenneth Siu Ho Chok ${ }^{1}$, Rex Kwok Him Au-Yeung², Chung Mau Lo' \\ ${ }^{1}$ Department of Surgery, Queen Mary Hospital, The University of Hong Kong, Hong Kong, China \\ ${ }^{2}$ Department of Pathology, Queen Mary Hospital, The University of Hong Kong, Hong Kong, China
}

\begin{abstract}
Hepatic sarcomatoid carcinomas are very rare. The majority of cases contain sarcomatoid features with either hepatocellular carcinoma (HCC) or cholangiocarcinoma (CC) elements alone. These are aggressive tumors and carry an unfavorable prognosis. We describe an extremely rare tumor sub-type of combined sarcomatoid $\mathrm{HCC}$ and $\mathrm{CC}$ in a hepatitis $\mathrm{B}$ virus carrier presenting with abdominal pain. Pre-operative imaging suggested a segment VI hepatocellular cancer with no metastatic spread. En bloc surgical resection with the right adrenal gland, Gerota's fascia and right hemidiaphragm was performed. The patient suffered early peritoneal tumor recurrence and lymph node metastasis. Pre-operative diagnosis of such sarcomatoid tumors is difficult. Current evidence for adjuvant treatment is also limited. Prognosis of these patients remains extremely poor, and surgery appears to be the only curative option in cases of early disease. It is essential that clinicians carry a high index of suspicion and awareness of this rare pathological entity to improve patient outcome.
\end{abstract}

Key words: Cholangiocarcinoma; hepatocellular carcinoma; sarcomatoid; spindle cell sarcoma

Address for correspondence:

Dr. Kenneth Siu Ho Chok, Division of Hepatobiliary and Pancreatic Surgery and Liver Transplantation, Department of Surgery, Queen Mary Hospital, The University of Hong Kong, Hong Kong, China. E-mail: kennethchok@gmail.com

Received: 18-01-2015, Accepted: 13-03-2015

\section{INTRODUCTION}

Hepatic sarcomatoid carcinomas are very rare. The reported incidence is $<10 \%$ with most cases only identified at autopsy. Diagnosis after surgical resection has been found in $1.8 \%$ of cases. ${ }^{[1]}$ Most patients are reported as individual case reports or small series only. There is a confusing and varied pathological terminology including carcinosarcoma, sarcomatoid carcinoma and spindle cell carcinoma, ${ }^{[1-3]}$ all likely to describe the same pathology. The majority of cases contain sarcomatoid features together with either hepatocellular carcinoma (HCC) or cholangiocarcinoma (CC) elements alone.

\begin{tabular}{|l|c|}
\hline \multicolumn{2}{|c|}{ Access this article online } \\
\hline \multirow{2}{*}{ Website: } & Quick Response Code \\
http://www.hrjournal.net/ & \\
& \\
DOI: & \\
10.4103/2394-5079.154331 & \\
&
\end{tabular}

A sarcomatous tumor with both HCC and CC components is extremely rare. These tumors have an aggressive behavior and are associated with poor prognosis. Management options are currently unclear and limited. We report a case of combined sarcomatoid HCC and CC with a literature review of reported cases based on their characteristics and treatment options.

\section{CASE REPORT}

A 69-year-old Asian man presented with right-sided abdominal pain. He was a known hepatitis B virus (HBV) carrier but otherwise had no significant past medical history. He had no regular follow-up and was not on anti-viral therapy. Physical examination was unremarkable. Laboratory investigations were consistent with Child's A liver cirrhosis. Liver function tests were normal apart from a raised alkaline phosphatase level at $168 \mu \mathrm{mol} / \mathrm{L}$. Alpha-feto protein (AFP) level was raised to $21 \mathrm{ng} / \mathrm{mL}$. Antibody to hepatitis e antigen ( $\mathrm{HBeAg}$ ) was positive whereas $\mathrm{HBeAg}$ and anti-hepatitis $C$ virus were negative. HBV DNA level was $178 \mathrm{IU} / \mathrm{mL}$. Computed tomography (CT) identified a 
cirrhotic liver with a $5.9 \mathrm{~cm} \times 5.7 \mathrm{~cm}$ irregularly shaped mass with heterogeneous contrast enhancement and contrast washout in portovenous phase arising from segment $\mathrm{VI}$ with suspected invasion into the postero-lateral chest wall [Figures 1 and 2]. Incidental bilateral renal cysts were also identified. Dual tracer positron emission tomography (PET) CT scan confirmed a segment VI lesion with predominantly 18F-fluorodeoxyglucose uptake (SUVmax 14.2), suggestive of a moderate to poorly differentiated HCC [Figure 3]. There was another discrete hypermetabolic ${ }^{11} \mathrm{C}$-acetate avid only lesion (SUVmax 9.8) in segment IV/NIII consistent with multifocal HCC. There was no evidence of metastatic spread. Left lobe liver volumetry was measured at $34.5 \%$ of estimated standard liver volume.

We proceeded to a laparotomy with a plan for curative resection. A large tumor was seen in the right subphrenic space with invasion into at least $50 \%$ of the right hemidiaphragm and segment $\mathrm{VI}$ of the liver. Intra-operatively it was difficult to determine whether the tumor was hepatic in origin [Figure 4]. Dense adhesions were also identified around the right adrenal gland and Gerota's fascia. The liver was severely cirrhotic with numerous regeneration nodules. Intra-operative ultrasound

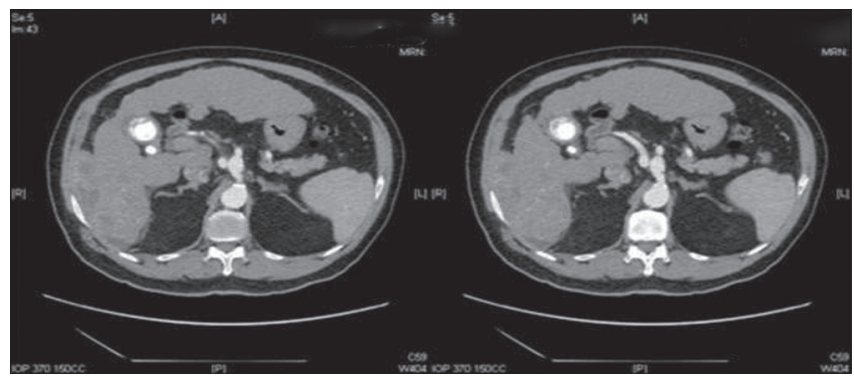

Figure 1: Computed tomography scan in arterial phase showing a segment $\mathrm{VI}$ heterogeneously enhancing mass with invasion to the postero-lateral chest wall

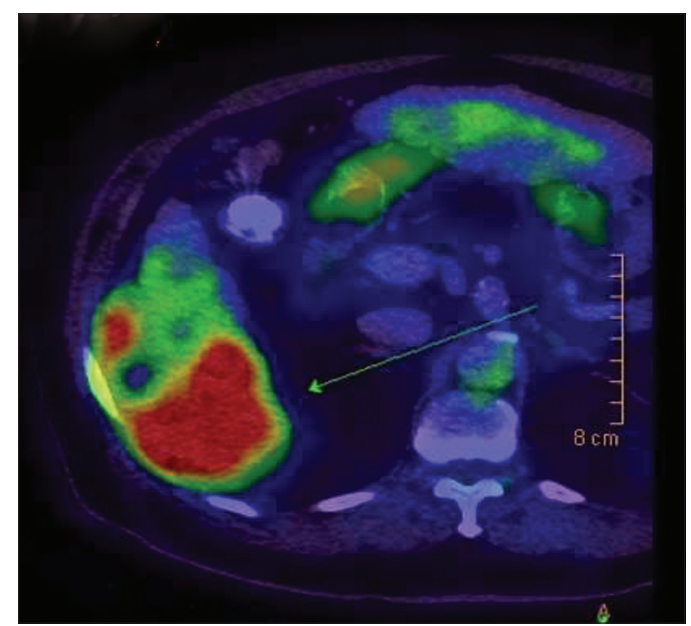

Figure 3: Positron emission tomography/computed tomography showing a predominantly $18 \mathrm{~F}$-fluorodeoxyglucose-avid liver mass suggestive of moderately to poorly differentiated hepatocellular carcinoma (SUVmax 14.2) could not identify any lesions at segment IV/NIII. A segment VI resection was performed together with en bloc resection of the right adrenal gland, upper Gerota's fascia and the invaded area of the right hemidiaphragm. The diaphragmatic defect was closed primarily with nylon sutures and reinforced with polytetrafluoroethylene mesh. Diaphragmatic satellite nodule frozen section suggested probable high-grade malignant tumor, but the origin could not be determined. Radiofrequency ablation was applied to the resection margins. Post-operative recovery was uneventful, and the patient was discharged on day 6. On follow-up CT scan 1-month after the operation, peritoneal nodularities up to $1.5 \mathrm{~cm}$ were identified. PET/CT scan confirmed metastatic deposits at the diaphragmatic mesh $(2.7 \mathrm{~cm}$, SUVmax 14) in addition to nodal deposits in the paracaval $(2.8 \mathrm{~cm}$, SUVmax 12.6) and pre-aortic regions (1.8 cm, SUVmax 9.6). The patient declined palliative chemotherapy and is currently receiving symptomatic care.

Pathological examination of the resected specimen revealed a $10 \mathrm{~cm} \times 9 \mathrm{~cm} \times 7 \mathrm{~cm}$ firm tan-colored tumor with a pushing margin, invading into diaphragmatic skeletal muscle [Figure $5 \mathrm{a}$ and $\mathrm{b}$ ]. The background liver showed features consistent with cirrhosis. Hepatitis B surface antigen was positive. Majority of the tumor was composed of

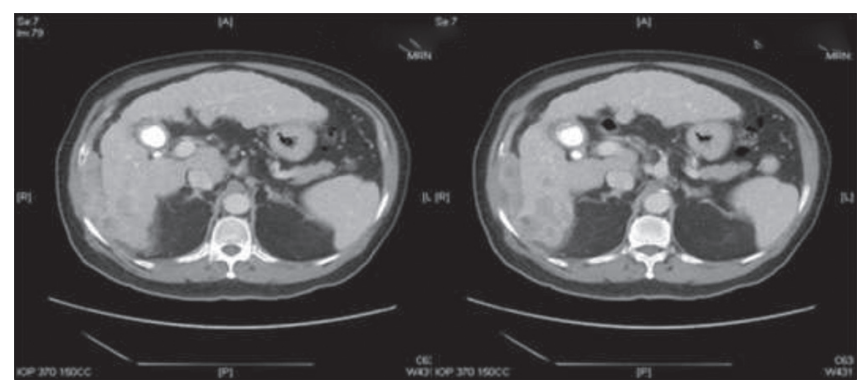

Figure 2: Computed tomography scan in portovenous phase showing a segment VI mass with contrast washout

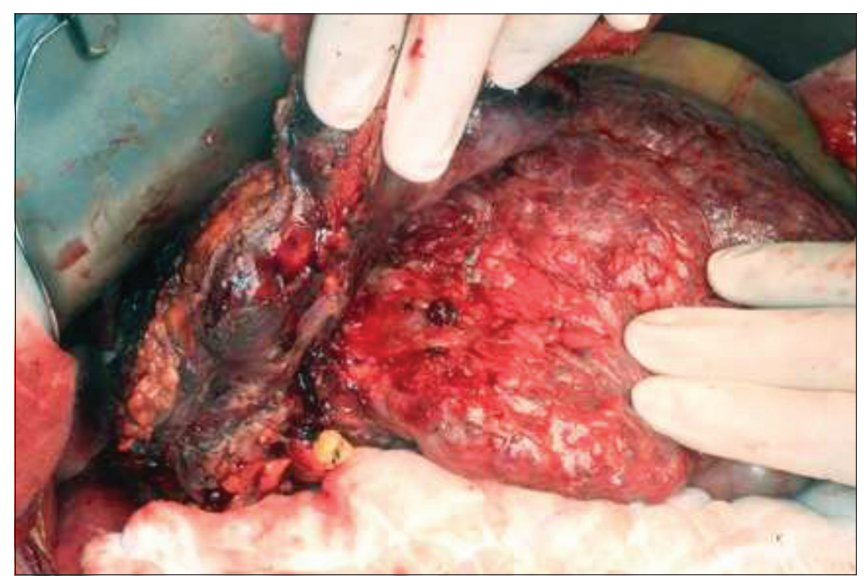

Figure 4: Intra-operative photo showing a cirrhotic liver with a segment VI mass invading into the right hemi-diaphragm and chest wall 


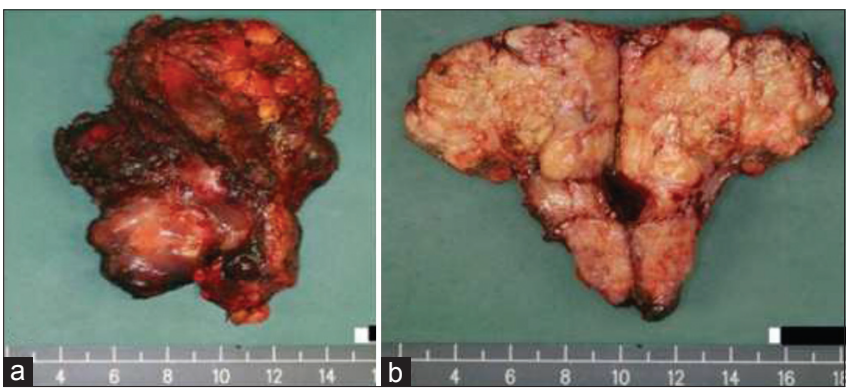

Figure 5: (a) Macroscopic appearance of the tumor and (b) gut section showing a tan-colored tumor with a pushing border

epithelioid to spindle-shaped cells with moderate cellularity. Other parts of the tumor composed of closely packed oval cells with high nuclear-cytoplasmic ratio and occasional acinar formation [Figure 6a]. Some parts of the tumor also composed of pleomorphic polygonal and multinucleated cells. The cells displayed moderate to severe nuclear pleomorphism and large patches of necrosis. Immunohistochemical staining showed diffusely strong membranous staining for pan-cytokeratin (CK) MNF1 16 (monoclonal antibody for carcinoma), low molecular weight CK CAM5.2 (monoclonal antibody for carcinoma), focal strong membranous staining for CK19 (CK19, monoclonal antibody for $\mathrm{CC}$ detection) and weak to moderate positivity for hepatocyte paraffin-1 (HEP-PAR-1, monoclonal antibody for hepatocyte detection for HCC). In areas with acinar formation, dot-like staining of CK7 (CK7, monoclonal antibody for bile duct differentiation) and CK19 was noted in apical parts of the cells toward the lumen. The overall features were consistent with a sarcomatoid carcinoma consisting of both hepatocellular (HEP-PAR-1 positivity) and CC (CK7 and CK19 positivity) differentiation [Figure $6 \mathrm{~b}$ and $\mathrm{c}$ ]. Final pathological staging was pT4 (American Joint Committee on Cancer, 7th edition).

\section{DISCUSSION}

Liver sarcomatoid carcinoma is a rare pathological entity. This highly malignant tumor usually contains an epithelial (hepatocellular or $\mathrm{CC}$ ) element together with sarcomatous mesenchymal cells. Less than 100 such sarcomatoid cases have been reported in the literature based on either a hepatocellular or CC element. The case reported here of combined sarcomatoid HCC, and CC is extremely rare and only a few such cases have been reported [Table 1].

Although the published nomenclature is inconsistent, they share common pathological features. As found in the present patient's tumor, large area of central necrosis is a characteristic feature of hepatic sarcomatoid carcinoma. The rapidly dividing sarcomatoid cells outgrow the neovasculature of the tumor, resulting in necrosis. ${ }^{[1]}$ Microscopically,

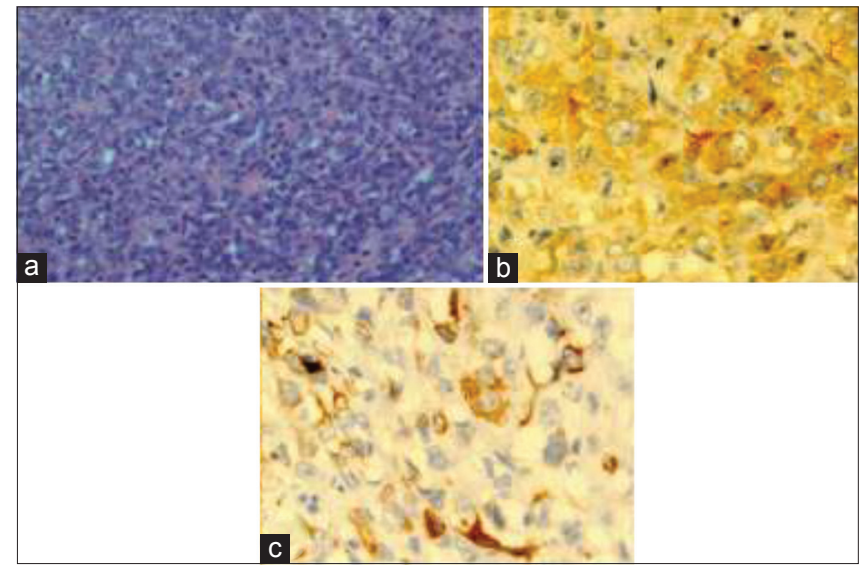

Figure 6: (a) Hematoxylin and Eosin slide of the tumor $(\times 400)$, and (b) tumor slide showing hepatocellular differentiation, hepatocyte paraffin-1 positive $(\times 400)$, and (c) bile duct differentiation, cytokeratin 19 positive $(\times 400)$

malignant epithelioid cells of the liver are found together with pleomorphic spindle-cells diagnostic of sarcomatous change. The pathogenesis is unclear but most believe that these tumors are as a result of either a differentiation of totipotential stem cells into mesenchymal cells and epithelial cells or the transformation of HCC or CC cells undergoing metaplasia into sarcomatous cells. ${ }^{[2]} \mathrm{A}$ transitional phenomenon has been observed in previous reports. Kakizoe et al. ${ }^{[10]}$ identified positive immunohistochemical staining of AFP and CK in sarcomatous cells suggesting the presence of a cell type transformation. In addition, the sarcomatous component could further differentiate into specialized cell types including rhabdomyoblastic, ${ }^{[1]}$ chondroid ${ }^{[12]}$ and hepatoblastoma-like. ${ }^{[13]}$

No definite identifiable risk factor for hepatic sarcomatoid carcinoma exists so far. Previous series reported an approximately $50 \% \mathrm{HBV}$ infection rate in these tumors. There is however, no evidence to suggest $\mathrm{HBV}$ infection is associated with an increased risk of their development. It has been suggested that previous cancer treatment including systemic target therapy and trans-arterial chemoembolization (TACE) may increase the risk of developing these tumors. ${ }^{[14]}$ Kojiro et al. ${ }^{[15]}$ reported that previous anti-cancer therapy such as TACE might pre-dispose HCC cells to undergo a metaplastic change into sarcomatoid cells. They observed a higher incidence of sarcomatoid carcinomas in those treated with TACE.

Clinically, these tumors pose a diagnostic challenge pre-operatively as they resemble HCCs in presentation. Their behavior however is much more aggressive than ordinary HCC. The diagnosis of a sarcomatoid element prior to pathological specimen examination has proven difficult. As in the present patient, most would initially be considered 
Table 1: Case reports of combined sarcomatoid hepatocellular carcinoma and cholangiocarcinoma

\begin{tabular}{|c|c|c|c|c|c|}
\hline Study & Age, gender & Symptoms & Hepatitis serology & Treatment & Follow-up and prognosis \\
\hline Nakajima et al. ${ }^{[4]}$ & 74 years, male & Right upper quadrant pain & $\mathrm{HBsAb}$ & TACE & $\begin{array}{l}\text { Lung metastasis; death at } 17 \text { months; } \\
\text { diagnosis at autopsy }\end{array}$ \\
\hline Papotti et al. ${ }^{[5]}$ & 59 years, male & Lumbar pain & Negative & Hepatectomy & Biliary fistula; death at 4 months \\
\hline Jeong et al. ${ }^{[6]}$ & 60 years, female & Right upper quadrant pain/mass & NA & Hepatectomy & Tumor recurrence; death at 12 months \\
\hline Kim et al. ${ }^{[7]}$ & 67 years, male & Synchronous colon tumor & NA & NA & NA \\
\hline Boonsakan et al. ${ }^{[8]}$ & 28 years, male & Fever & $\mathrm{HBsAg}$ & Palliative resection & Lost to follow-up \\
\hline
\end{tabular}

NA: not available; HBsAb: hepatitis B surface antibody; HBsAg: hepatitis B surface antigen; TACE: trans-arterial chemoembolization

ordinary HCC with positive hepatitis serology, cirrhosis and raised AFP levels. Liver function parameters may or may not be unstable depending on the size of the tumor and degree of underlying cirrhosis. The liver was grossly cirrhotic [Figure 4] in our patient; however, his child's score was only Class A. This might be attributed to his relatively large liver volume reserve compensating for cirrhosis. Some authors have reported high fever and abdominal pain as frequent symptoms of sarcomatoid HCC. ${ }^{[13,16]}$ Although these were not present in this patient, the suspicion of liver abscess based on these symptoms may delay and make the diagnosis more difficult. In a patient described by Inoue et al., ${ }^{[13]}$ abscess drainage followed by biopsy were performed before the diagnosis was made. At 3 weeks after admission, there was already pleural metastasis. Despite a trial of chemotherapy, the patient died of multiorgan failure on day 27. Radiologically, our patient's tumor was also suggestive of HCC with arterial enhancement and portovenous contrast washout. Honda et al..$^{[17]}$ described delayed or prolonged contrast enhancement as a feature of sarcomatoid HCC. This finding may be attributed to the presence of active cancerous tissue with fibrous stroma. The aggressiveness of this type of tumor is highlighted by the high incidence of local invasion and metastatic spread either at the time of surgery or early recurrence. Indeed, there was invasion into the right hemidiaphragm of $>50 \%$ in our patient. Both intra and extrahepatic metastasis as well as lymphadenopathy is common. ${ }^{[16]}$ Metastasis to multiple organs including the lungs, hepatic hilar lymph nodes, greater omentum, stomach and diaphragm as seen in our patient have been reported. ${ }^{[4]}$

Prognosis is inevitably poor with such aggressive behavior of these tumors with high risk of metastasis. In the cases reported in the literature, most patients have developed metastatic lesions within 3-6 months of diagnosis and survival rarely exceeded 12 months. This is exemplified in the present patient, where metastatic lymph nodes and peritoneal nodules were already identified 1-month post-surgical resection. In a study reported by Aishima et al., ${ }^{[18]} 7$ (17.5\%) out of 40 patients had a sarcomatous component in combined HCC and CC. The rest of the patients were divided according to the percentage of CC. Sarcomatous patients were found to have significantly higher chance of vascular invasion, lymph node metastasis as well as high CC percentage group, and were associated with a much shorter survival compared to those without a sarcomatous and lower CC component $(P<0.0048)$. Such aggressive lesions make the diagnosis and treatment difficult. Patients are either diagnosed only at post-mortem or at an advanced unresectable stage. There is currently no recommended treatment strategy for sarcomatoid carcinomas, and surgical resection appears to be the only curative option, albeit only at the early stage of tumor formation. There is yet no report of successful adjuvant treatment with chemotherapy or radiotherapy described in the literature to prolong survival in patients with this aggressive tumor.

In conclusion, a case of hepatic combined sarcomatoid HCC and CC is reported here. This is an extremely rare tumor with an aggressive behavior. The prognosis is extremely poor, and survival has been limited to within a few months in most cases. Pre-operative diagnosis and distinction from ordinary HCC is difficult. Reports in the literature suggest surgery as the treatment of choice at present. Due to the rarity of the disease, adjuvant chemotherapy or radiotherapy has not shown any promise. Early diagnosis with a high index of suspicion appears to give the best chance of cure.

\section{REFERENCES}

1. Koo HR, Park MS, Kim MJ, Lim JS, Yu JS, Jin H, Kim KW. Radiological and clinical features of sarcomatoid hepatocellular carcinoma in 11 cases. J Comput Assist Tomogr 2008;32:745-9.

2. Lao XM, Chen DY, Zhang YQ, Xiang J, Guo RP, Lin XJ, Li JQ. Primary carcinosarcoma of the liver: clinicopathologic features of 5 cases and a review of the literature. Am J Surg Pathol 2007;31:817-26.

3. Maeda T, Adachi E, Kajiyama K, Takenaka K, Sugimachi K, Tsuneyoshi M. Spindle cell hepatocellular carcinoma. A clinicopathologic and immunohistochemical analysis of 15 cases. Cancer 1996;77:51-7.

4. Nakajima T, Kubosawa H, Kondo Y, Konno A, Iwama S. Combined hepatocellular-cholangiocarcinoma with variable sarcomatous transformation. Am J Clin Pathol 1988;90:309-12.

5. Papotti M, Sambataro D, Marchesa P, Negro F. A combined hepatocellular/cholangiocellular carcinoma with sarcomatoid features. 
Liver 1997; 17:47-52

6. Jeong BJ, Hyun DH, Lee KW, Ryu ST, Lee JW, Lee JI, Jeong S, Lee DH, Kim PS, Kim HG, Kim YS, Kim JM. A case of sarcomatoid combined hepatocellular-cholangiocarcinoma. Korean J Gastroenterol 2004;43:56-60.

7. Kim JH, Lee YG, Lee J, Jung CK, Kim HT, Kang H, Kho KH, Hong SP, Hwang SG, Park PW, Rim GS. A case of combined hepatocellular-cholangiocarcinoma with sarcomatous transformation and second primary colon cancer. Korean J Hepatol 2004;10:142-7.

8. Boonsakan P, Thangnapakorn O, Tapaneeyakorn J, Kositchaiwat S, Bunyaratvej S. Case report combined hepatocellular and cholangiocarcinoma with sarcomatous transformation. J Med Assoc Thai 2007;90:574-80

9. Pua U, Low SC, Tan YM, Lim KH. Combined hepatocellular and cholangiocarcinoma with sarcomatoid transformation: radiologic-pathologic correlation of a case. Hepatol Int 2009;3:587-92.

10. Kakizoe S, Kojiro M, Nakashima T. Hepatocellular carcinoma with sarcomatous change. Clinicopathologic and immunohistochemical studies of 14 autopsy cases. Cancer 1987;59:310-6.

11. Akasofu M, Kawahara E, Kaji K, Nakanishi I. Sarcomatoid hepatocellular-carcinoma showing rhabdomyoblastic differentiation in the adult cirrhotic liver. Virchows Arch 1999;434:511-5.

12. Fu Y, Kobayashi S, Kushida Y, Saoo K, Haba R, Mori S, Miki H, Ohmori M. Sarcomatoid hepatocellular carcinoma with chondroid variant: case report with immunohistochemical findings. Pathol Int 2000;50:919-22.

13. Inoue T, Kudo M, Minami Y, Chung H, Fukunaga T, Kawasaki T. Case of rapidly progressed sarcomatoid hepatocellular carcinoma in a young female without risk factor. Liver Int 2007;27:1428-30.

14. Marijon H, Dokmak S, Paradis V, Zappa M, Bieche I, Bouattour M, Raymond E, Faivre S. Epithelial-to-mesenchymal transition and acquired resistance to sunitinib in a patient with hepatocellular carcinoma. J Hepatol 2011;54:1073-8.

15. Kojiro M, Sugihara S, Kakizoe S, Nakashima O, Kiyomatsu K. Hepatocellular carcinoma with sarcomatous change: a special reference to the relationship with anticancer therapy. Cancer Chemother Pharmacol 1989;23 Suppl:S4-8.

16. Eriguchi N, Aoyagi S, Okuda K, Hara M, Fukuda S, Tamae T, Ohdo M, Kanazawa N, Kawabata M, Kodama T, Nishimura K, Hamada S. Unusual liver carcinomas with sarcomatous features: analysis of four cases. Surg Today 2001;31:530-3.

17. Honda H, Hayashi T, Yoshida K, Takenaka K, Kaneko K, Fukuya T, Tateshi Y, Ro T, Maeda T, Masuda K. Hepatocellular carcinoma with sarcomatous change: characteristic findings of two-phased incremental CT. Abdom Imaging 1996;21:37-40.

18. Aishima S, Kuroda Y, Asayama Y, Taguchi K, Nishihara Y, Taketomi A, Tsuneyoshi M. Prognostic impact of cholangiocellular and sarcomatous components in combined hepatocellular and cholangiocarcinoma. Hum Pathol 2006;37:283-91.

How to cite this article: Tsang JS, Chok KS, Au-Yeung RK, Lo CM. Combined sarcomatoid hepatocellular and cholangiocarcinoma: a case report and literature review. Hepatoma Res 2015;1:41-5.

Source of Support: Nil. Conflict of Interest: None declared. 